\title{
Effect of user puffing topography on total particulate matter, nicotine and volatile carbonyl emissions from narghile waterpipes
}

\author{
Nathan C Eddingsaas (1) , ${ }^{1}$ Edward C Hensel, ${ }^{2}$ Sean O'Dea, ${ }^{1}$ Peyton Kunselman, ${ }^{1}$ \\ A Gary DiFrancesco, ${ }^{2}$ Risa J Robinson ${ }^{2}$
}

${ }^{1}$ School of Chemistry and Materials Science, College of Science, Rochester Institute of Technology, Rochester, New York, USA

${ }^{2}$ Department of Mechanical Engineering, Kate Gleason College of Engineering, Rochester Institute of Technology, Rochester, New York, USA

\section{Correspondence to} Dr Nathan C Eddingsaas, Chemistry, Rochester Institute of Technology, Rochester, NY 14623, USA; ncesch@rit.edu

Received 24 January 2019 Revised 7 July 2019

Accepted 9 July 2019 Published Online First 12 August 2019
Check for updates

(C) Author(s) (or their employer(s)) 2020. No commercial re-use. See rights and permissions. Published by BMJ.

To cite: Eddingsaas NC, Hensel EC, O'Dea S, et al. Tob Control

2020;29:s117-s122.

\section{ABSTRACT}

Objectives Puffing topographies of waterpipe users vary widely as does the puff-to-puff topography of an individual user. The aim of this study was to determine if puff duration and flow rate have an effect on the characteristics of the mainstream emission from waterpipes, including total particulate matter (TPM), mass ratio of nicotine and mass concentration of volatile carbonyls.

Methods Puffing parameters were chosen to encompass a significant portion of the perimeter space observed from a natural environment study. Tested conditions were 150, 200 and $250 \mathrm{~mL} \mathrm{sec}^{-1}$; each run at 2, 3.5 and $5 \mathrm{~s}$ durations; 25 s interpuff duration and $\sim 100$ puffs per session. Each session was run in quadruplicate using the Programmable Emissions System-2 (PES-2) emissions capture system under identical conditions. Particulate matter, for quantification of TPM and nicotine, was collected on filter pads every $\sim 5 \mathrm{~L}$ of aerosol resulting in 6 to 25 samples per session. Volatile carbonyls were sampled using 2,4-Dinitrophenylhydrazine (DNPH)-coated silica.

Results Mass concentration of TPM linearly decreased with increased flow rate, with no dependency on puff duration. Nicotine mass ratio was independent of topography, with average mass ratio of nicotine to TPM of $0.0027 \pm 0.0002(\mathrm{mg} / \mathrm{mg})$. The main carbonyls observed were acetaldehyde and formaldehyde. Puff duration increased emissions of some carbonyls (eg, formaldehyde) but not others (eg, acetaldehyde).

Conclusions The results presented here highlight that topographies influence the emissions generated from waterpipes including TPM, total nicotine and volatile carbonyls. For laboratory studies to be representative of user exposure, a range of topographies must be studied. Using a range of topographies within a controlled laboratory environment will better inform regulatory policy.

\section{INTRODUCTION}

Narghile waterpipe, also known as hookah, is an ancient form of nicotine delivery that has seen a resurgence in the past several years. ${ }^{1}$ With increased use comes more need to understand human exposure and increased regulation for better health outcomes. To this end, an understanding of the baseline emissions associated with hookah smoking is needed to determine the parameters that increase emissions, and a benchmark to determine the effect of regulation or other changes. Over the years researchers have worked to determine the emissions from smoking hookah, measuring many parameters including particulate matter, nicotine, volatile carbonyls and many others. ${ }^{2-9}$ While these measurements provide the emissions from hookah in a laboratory setting, the vast majority of the studies have used just one puffing topography: 171 puffs, $2.6 \mathrm{~s}$ puffs, $208 \mathrm{~mL} \mathrm{~s}^{-1}$ flow rate and $17 \mathrm{~s}$ between puffs. This protocol was first designed based off of the averages from observations in a hookah bar. ${ }^{10} 11$ By using this protocol, results can be compared between laboratories, but it is not representative of the full range of how people smoke a hookah. Topographies vary user-to-user and for a given user session-to-session and even puff-to-puff. ${ }^{12-14}$

We recently conducted a natural environment puffing topography study, where users used a Rochester Institute of Technology (RIT) wireless personal use monitor (wPUM) hookah monitor in their own residential settings for 1 week. Preliminary results show that average puffing topographies vary personto-person with average puff flow rates varying from 82 to $389 \mathrm{~mL} \mathrm{sec}{ }^{-1}$ with a mean of $245 \mathrm{~mL} \mathrm{sec}{ }^{-1}$, and average puff durations varying between 1.9 and $5.8 \mathrm{~s}$ with an average of $3.5 \mathrm{~s}$. In addition, session-tosession and puff-to-puff variations in topographies were observed from all users. ${ }^{15}$ Other researchers have also reported variation in user topographies, but to date, no laboratory studies have been conducted to determine the effect of topography on emissions. ${ }^{14} 16$ A recent study, where emissions were collected while users smoked a waterpipe, showed that users inhaled a large amount of toxicants, greater than what would be suggested by laboratory studies. ${ }^{17}$ This indicates that current laboratory studies using one puffing topography do not represent real world use of waterpipes, where variation of the topography is the norm.

In this study, we evaluate the effect that two variables of topography, puff duration and puff flow rate, have on emissions of particulate matter, nicotine and volatile aldehydes. To obtain these results, we studied nine topographies within the range of typical user topographies using RIT's programmable emissions system (PES-2) smoking machine, comparing results across topographies while normalising the results to either puff volume of mass of particulate matter produced.

\section{MATERIALS AND METHODS Hookah smoking machine and emissions generation and sampling}

Hookah product characteristics were held constant so that the effect of puffing topography could be analysed. A commercial hookah (Elite) equipped with a Budpro Vortex Hookah Bowl was used for all trials. Eighteen-hundred millilitres of fresh water was 
added to the hookah base prior to each trial so that the downstem was submerged $38 \pm 6 \mathrm{~mm}$ into the water and $10 \mathrm{~g}$ of 'Two Apple' flavoured shisha (Al Fakher) was added to the bowl and covered by foil with holes patterned after Shihadeh et al. ${ }^{10}$ The amount of shisha used was in excess to ensure reproducibility. One instant light charcoal (Starlight) was lit away from the hookah and allowed to fully ignite prior to being placed on top of the foil. Each trial was initiated $160 \mathrm{~s}$ after placement of the coal on the foil. A trial lasted approximately $50 \mathrm{~min}$, regardless of puffing parameters. After a trial, the remaining shisha was weighed and then discarded, the apparatus was allowed to cool and then the bowl was washed and scrubbed using warm water. The base was emptied and rinsed with warm water after each trial. The shaft and hose were washed periodically when enough time could be afforded for complete drying.

Quality control trials were performed to ensure the results observed were from the variation in topography and not from product characteristic or environmental conditions. Two trials were performed in the same manner as all other trials with one component removed. The first had all components assembled besides the coal, and puffing was performed per one of the parameters discussed below. A second test trial performed was with the coal but no shisha. Reproducibility of results was also confirmed by semi-random order of trials over two time blocks. Two trials at each topography were performed between 7 April, 2018, and 27 April, 2018, and two more trials of each parameter were run between June $11^{\text {th }}$ and June $27^{\text {th }}$, in different order. Results were not statistically different at a given set of puffing topographies, and all trials were averaged.

Trials were performed using an in-house mechanical puffing machine, PES-2. The RIT programmable emissions system, PES-2, used for this study is a higher flow rate variant of the PES-1 machine puffing system previously reported and described for use with electronic cigarettes. ${ }^{18}$ The PES-2 employs third-party-calibrated flow metres to measure the transient flow rate through the system immediately downstream from the filter collection pad. The nominal puff durations of 2, 3.5 and $5 \mathrm{~s}$ and puff flow rates of 150, 200 and $250 \mathrm{~mL} \mathrm{sec}^{-1}$ are used for grouping trials and labelling the figures and are presented in table 1 for organising the results of repeated trials. In addition, table 1 includes mean and SD in observed flow rates and durations for each of the nominal conditions. The observed mean puff flow rates, puff duration and puff volume are used for plotting the discrete points in figures 1-3 and were determined from the measured quantities using the Topography Analysis Program (TAP) previously described. ${ }^{19-22}$ TAP computes the mean value, SD and $95 \%$ CIs on all topography parameters. The PES-2 was designed to accommodate tobacco products with flow rates ranging from 10 to $350 \mathrm{~mL} \mathrm{sec}^{-1}$ and has been used for testing

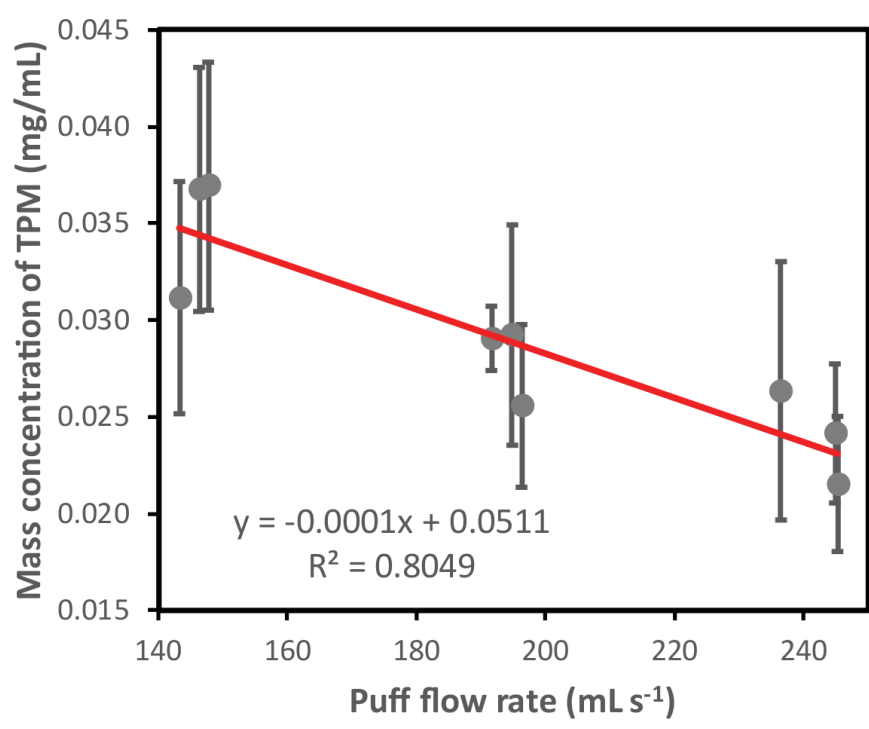

Figure 1 Concentration of total particulate matter ( $\mathrm{mg}$ of particulate matter per $\mathrm{mL}$ of aerosol) as a function of puff flow rate. All nine puffing topographies are shown independently. Error bars represent one SD of repeat trials. A trend of TPM concentration is observed as a function of puff flow rate, but not as a function of puff duration (not shown). TPM, total particulate matter.

waterpipes and waterpipe components as previously reported..$^{23-25}$ Particulate emissions were collected on a Cambridge style filter pad directly after the output of the hookah mouthpiece. PES-2 also includes the ability to capture volatile emissions including volatile carbonyls and volatile organic compounds (VOCs) with the use of sorption tubes (LpDNPH S10 (Supelco 21024U) and ORBO-43 (Supelco 20258)). These emissions were captured via side stream sampling with flow rates limited to under $30 \mathrm{~mL} \mathrm{sec}^{-1}$ by critical orifices manufactured by the O'Keefe Controls Co.

Puffing parameters were chosen to encompass a representative perimeter space observed from a natural environment study performed by our laboratory. A natural environment study was in progress at the time the current emissions study was initiated. Preliminary observations of waterpipe users $(n=17)$ in their natural environment showed a mean puff flow rate of $223 \mathrm{~mL} \mathrm{sec}$ ${ }^{1}$, duration of $2.5 \mathrm{~s}$, puff count of 109 puffs/session and session volume of $71.6 \mathrm{~L}^{24}$ Average topographies from this study are similar to previous natural environment studies where average puff durations range from 2.6 to $3 \mathrm{~s}$ and puff flow rates range from 203 to $272 \mathrm{~mL} \mathrm{sec}^{-1}$. ${ }^{1011} 1726$ Users were instructed to use

\begin{tabular}{|c|c|c|c|c|c|c|}
\hline \multirow[b]{2}{*}{ Sample ID } & \multicolumn{2}{|l|}{ Nominal } & \multicolumn{2}{|l|}{ Measured } & \multirow[b]{2}{*}{ \# puffs } & \multirow[b]{2}{*}{ Total volume (L } \\
\hline & Flow rate $\left(\mathrm{mL} \mathrm{sec}{ }^{-1}\right)$ & Puff duration (s) & Flow rate $\left(\mathrm{mL} \mathrm{sec}^{-1}\right)$ & Puff duration (s) & & \\
\hline $150 / 3.5$ & 150 & 3.5 & $146.3(4.4)$ & $3.70(0.02)$ & 100 & $54.2(1.4)$ \\
\hline $150 / 5$ & 150 & 5 & $147.7(3.2)$ & $5.20(0.01)$ & 105 & $80.6(1.7)$ \\
\hline $200 / 2$ & 200 & 2 & $191.7(7.9)$ & $2.21(0.01)$ & 104 & $44.1(1.8)$ \\
\hline $200 / 5$ & 200 & 5 & $196.3(3.3)$ & $5.21(0.00)$ & 100 & $102.4(1.8)$ \\
\hline $250 / 2$ & 250 & 2 & $236.5(9.8)$ & $2.24(0.03)$ & 100 & $52.9(1.7)$ \\
\hline $250 / 3.5$ & 250 & 3.5 & $245.3(6.0)$ & $3.70(0.02)$ & 102 & $92.7(1.8)$ \\
\hline $250 / 5$ & 250 & 5 & $244.9(5.0)$ & $5.23(0.03)$ & 100 & $128.0(1.9)$ \\
\hline
\end{tabular}

Measured flow rates and puff durations are average values of all puffs at a given topography. Values in parenthesis are one SD. 


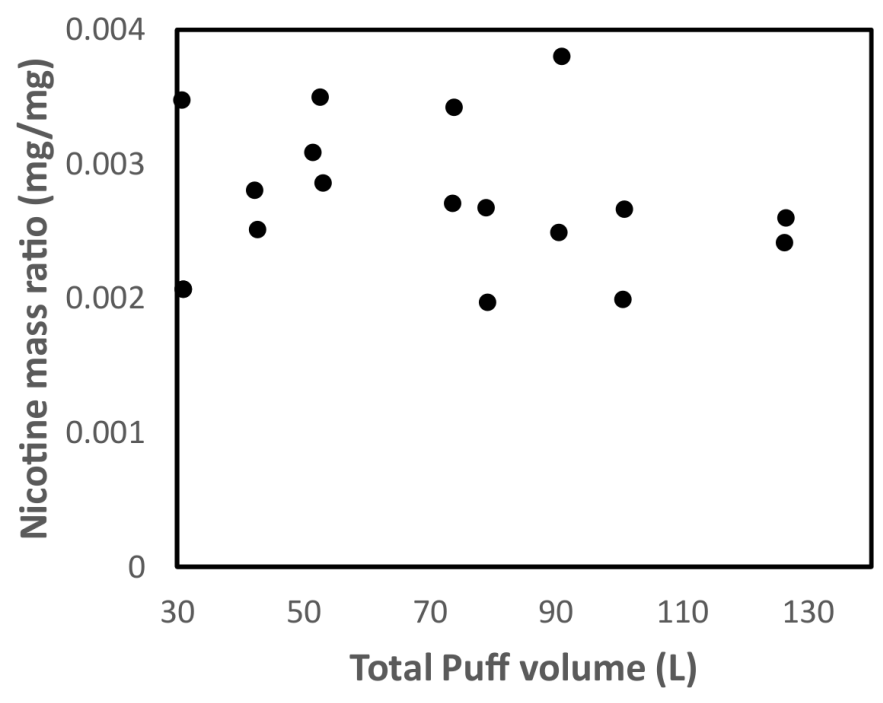

Figure 2 Nicotine mass ratio as a function of total session puff volume across all puffing topographies studied. All 18 trials with nicotine data, as shown in table 2, are shown. Nicotine mass ratio was found to be independent of both puff flow rate and puff duration, with nicotine mass ratio of $0.00272 \pm 0.00022$ ( $95 \% \mathrm{Cl}, 18$ samples).

their own-choice waterpipe, tobacco and flavours ad libitum as reflecting their normal use during a 1 week period. Subsequent analysis of all 7493 puffs taken by $n=24$ users during the natural environment study indicates the emissions study puff duration range of 2.5 to $5 \mathrm{~s}$ corresponds to approximately the $25^{\text {th }}$ to $85^{\text {th }}$ percentile of observed puff durations. Similarly, the emissions study puff flow rate range of 150 to $250 \mathrm{~mL} \mathrm{sec}^{-1}$ corresponds to approximately the $45^{\text {th }}$ to $80^{\text {th }}$ percentile of observed puff flow rates. Additional details on the natural environment topography of waterpipe users is beyond the scope of this article and will be reported separately. We chose to conduct four trials at each of nine flow conditions to assess the SD and 95\% CIs of results.

\section{Quantification of emissions}

Particulate matter was collected onto $44 \mathrm{~mm}$ silica Cambridge filter pads directly after it exited the mouthpiece. Mass of aerosol was determined gravimetrically for each pad immediately after

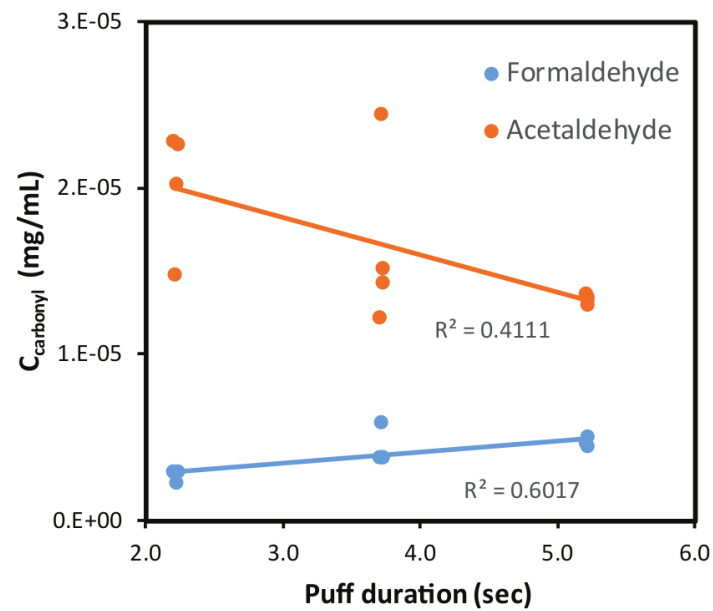

Figure 3 Mass concentration of formaldehyde and acetaldehyde at $200 \mathrm{~mL} \mathrm{sec}^{-1}$ as a function of puff duration. Similar trends of increasing formaldehyde and decreasing acetaldehyde as puff duration increase was observed at the other flow rates studied. removal from the filter holder. Tests were conducted to ensure complete sampling of particulate matter without any breakthrough by performing multiple smoking runs with two filters in individual holders in series; no breakthrough was observed.

Nicotine in the particulate matter was determined in the same manner as performed previously. ${ }^{27}$ In brief, pads were spiked with quinolone as an internal standard, submerged in methanol, shaken to break up the pads and filtered prior to analysis. Nicotine concentration was determined by gas chromatography mass spectrometry (GCMS) analysis on a Shimadzu QP2010 GCMS equipped with a AOC-20s autosampler using a protocol identical to that used previously. ${ }^{27}$

Volatile carbonyls were sampled using Supelco LpDNPH S10 cartridges. Flow rates through the tubes were always less than $2 \mathrm{slm}$. Quality control tests were conducted with two S10 cartridges sampling in series to determine if there was any loss of carbonyls using one tube. Minimal (never more than $0.5 \%$ ) to no carbonyls were detected on the second tube. Carbonyls were extracted with $2 \mathrm{~mL}$ of acetonitrile, diluted to $5 \mathrm{~mL}$ and quantified byliquid chromatography mass spectrometry (LCMS) using a Shimadzu 2020 LCMS. Samples were injected into the LC using an auto sampler in $5 \mu \mathrm{L}$ aliquots and eluted at a flow rate of $0.600 \mathrm{~mL} \mathrm{~min}^{-1}$ through a Shimadzu $50 \mathrm{~mm} \mathrm{C18} \mathrm{column}$. The mobile phase gradient started at $50 \%$ of $0.10 \%$ formic acid and $50 \%$ acetonitrile for $3 \mathrm{~min}$ then ramping to $63 \%$ acetonitrile over the next $9 \mathrm{~min}$ and finally a spike to $90 \%$ acetonitrile for $4 \mathrm{~min}$. Derivatized carbonyls were detected and quantified using mass spectrometry and ultraviolet-visible absorption. Mass spectral analysis was done using single ion monitoring for each analyte; m/z 209, 223, 235, 237, 251 and 285 for DNPHderivatized formaldehyde, acetaldehyde, acrolein, acetone and propionaldehyde (both at $\mathrm{m} / \mathrm{z} 237$ ), butyraldehyde and benzaldehyde, respectively. All derivatized carbonyls were also detected and analysed by absorption at $403 \mathrm{~nm}$. All standards and samples were run in triplicate to ensure reproducibility of the analysis procedure.

\section{RESULTS}

A total of 37 laboratory hookah smoking sessions across the nine topographies were conducted. The average emissions observed including total particulate matter, nicotine and carbonyls including formaldehyde, acetaldehyde, combined acetone and acrolein, propionaldehyde and butyraldehyde can be seen in table 2 . The mass concentration of total particulate matter and individual carbonyls ( $\mathrm{mg}$ of analyte to $\mathrm{mL}$ of air drawn through the hookah) as well as the mass ratio of nicotine (mg of nicotine to $\mathrm{mg}$ of particulate matter) are reported for comparison across topographies. Acetone and acrolein are reported as a combined value because they coeluted from the liquid chromatography column and the detection method, absorption spectroscopy, cannot separate them. The sum is valid because the absorption cross sections of the two derivatized carbonyl species are the same. The average values at each topography condition $(\mathrm{n}=4$ or 5$)$ are presented as well as the $\mathrm{SD}$ of the measurements in parentheses.

The total particulate matter produced was linearly correlated with total shisha consumed $\left(\mathrm{R}^{2}=0.77\right.$, data not presented) across all topographies tested. The mass concentration of total particulate matter as a function of puff flow rate is shown in figure 1 . Presented in figure 1 is the average mass concentration at each of the nine topographies tested as well as one SD of the results represented by the error bars. The concentration was calculated using the measured puff volume, rather than 
Table 2 Emissions generated from different hookah smoking topographies

\begin{tabular}{|c|c|c|c|c|c|c|c|}
\hline \multirow[b]{2}{*}{ Sample ID } & \multirow{2}{*}{$\begin{array}{l}\text { Particulate mass } \\
\text { concentration }(\mathrm{mg} / \\
\mathrm{mL})\end{array}$} & \multirow[b]{2}{*}{ Nicotine mass ratio $(\mathrm{mg} / \mathrm{g})$} & \multicolumn{5}{|c|}{ Mass concentration $(\mu \mathrm{g} / \mathrm{L})$ of carbonyls } \\
\hline & & & Form-aldehyde & $\begin{array}{l}\text { Acet- } \\
\text { aldehyde }\end{array}$ & $\begin{array}{l}\text { Acetone/ } \\
\text { acroline }\end{array}$ & $\begin{array}{l}\text { Propion- } \\
\text { aldehyde }\end{array}$ & Butyr-aldehyde \\
\hline $150 / 2$ & $0.031(0.007)$ & $2.06,2.07,3.47$ & $3.0(1.1)$ & $22(6)$ & $6.9(2.7)$ & $1.7(0.2)$ & $2.6(0.7)$ \\
\hline $150 / 3.5$ & $0.037(0.006)$ & $2.85,3.49$ & $2.9(0.3)$ & $18(4)$ & $6.8(1.4)$ & $1.4(0.3)$ & $1.8(0.5)$ \\
\hline $150 / 5$ & $0.037(0.007)$ & $1.96,2.67$ & $4.4(0.9)$ & $16(5)$ & $8.0(2.6)$ & $1.4(0.5)$ & $1.4(0.4)$ \\
\hline $200 / 2$ & $0.029(0.002)$ & $2.50,2.79$ & $2.7(0.3)$ & $20(4)$ & $5.9(1.2)$ & $1.4(0.2)$ & $2.1(0.5)$ \\
\hline $250 / 2$ & $0.026(0.007)$ & 3.07 & $4.2(2.2)$ & $25(13)$ & $10(5.4)$ & $2.5(1.6)$ & $2.2(0.9)$ \\
\hline $250 / 3.5$ & $0.022(0.004)$ & $3.79,2.48$ & $5.9(0.2)$ & $19(16)$ & $8.2(7.1)$ & $2.0(2.4)$ & $1.5(1.2)$ \\
\hline $250 / 5$ & $0.024(0.004)$ & $2.59,2.41$ & $7.1(1.3)$ & $14(5)$ & $7.2(3.0)$ & $1.2(0.7)$ & $1.2(0.4)$ \\
\hline
\end{tabular}

Data reported are average values at a given topography with the SD of the measurements in parentheses. The number of trials averaged for each topography was four except $150 \mathrm{~mL} \mathrm{sec}{ }^{-1}, 2 \mathrm{~s}$ where five trials were done. All values for nicotine mass ration are presented as a subset of trials were analysed for nicotine.

the nominal volume. The linear regression line is a fit through all data points; the individual puff durations are colour coded for clarity only.

Nicotine mass ratio as a function of total puff volume is displayed in figure 2. All puff topographies are included, the identification ofthe topography for each point can be determined from the data presented inTable 1.

The mass concentration and mass ratio of individual volatile carbonyls were analysed as a function of puffing topography. Figure 3 shows the mass concentration of two of the volatile carbonyls, formaldehyde and acetaldehyde, as a function of puff duration at $200 \mathrm{~mL} \mathrm{sec}^{-1}$. All of the individual trials are shown, four trials per topography. Similar results were observed for the other two puff flow rates.

\section{DISCUSSION}

Two puffing parameters were tested that were thought to have the largest influence on emissions from smoking of a hookah: puff flow rate and puff duration. Puff flow rate was found to have a large influence on mass concentration of particulate matter with a linear decrease from 150 to $250 \mathrm{~mL} \mathrm{sec}^{-}$ ${ }^{1}$. As shown in figure 1 , no dependency of puff duration on the particulate matter mass concentration was observed. The strong linear correlation between shisha consumed and total particulate matter across all topographies indicates that the change in mass concentration is not due to large changes in volatilisation of emissions but is more due to the process of aerosol production with less being formed at higher flow rates.

The session average nicotine mass ratio was found not to be affected by puff duration or puff flow rate. A subset of 18 of the 37 sessions had the mass of nicotine per filter pad analysed, and the results showed a spread of mass ratio but no systematic trend with either duration or flow rate. As the nicotine mass ratio per session was not dependent on topographies, the mass ratio can be computed using all 18 trials. The mass ratio of nicotine was found to be $0.00272 \pm 0.00022 \mathrm{mg} / \mathrm{mg}$ (95\% CI). This is similar to other nicotine mass ratios reported in the literature for hookah smoking; for examples, the mass ratio was found to be $0.0013,0.0022,0.00224,0.0026$ and 0.0048 by a number of studies by Shihadeh and coworkers, the last two being natural environment studies, and 0.0029 by Schubert et al..$^{249172628}$ It is not surprising that the mass ratio is slightly different in these different studies as the shisha used in each was different.
Seven carbonyl compounds were quantified from all 37 sessions in this study. The high priority volatile carbonyls sampled were formaldehyde, acetaldehyde and acrolein, known thermal decomposition products of the humectant. Table 2 shows the emissions of six of the carbonyls (benzaldehyde is not shown as it was not observed at concentrations greater than background). A smoking session that included all components except the shisha but including the lit coal was conducted, and all samples were collected for a background reference. The only emission with levels close to those with shisha present was benzaldehyde, where approximately the same levels were observed in the reference trial. Acrolein and acetone were left out of further analysis, as the concentration of each individually could not be determined here. As seen in figure 3 , as the puff duration increases, the mass concentration of formaldehyde increases and the mass concentration of acetaldehyde decreases. Similarly to acetaldehyde, the mass concentration of propionaldehyde and butyraldehyde were observed to decrease as puff duration increased. Conversely, no trend was observed when analysing the mass concentration of any of the carbonyls with respect to flow rate. Two previous studies have reported enough information to assess mass concentration of volatile carbonyls from smoking a hookah. ${ }^{39}$ Average formaldehyde was found to be $6.9 \times 10^{-6} \mathrm{mg} / \mathrm{mL}$ in Al Rashidi et al and $0.61-1.2 \times 10^{-7} \mathrm{mg} / \mathrm{mL}$ in Schubert et al. Our results compare well with Al Rashidi et al, ${ }^{3}$ and both Al Rashidi and ours are around an order of magnitude greater than those of Schubert et al. It is interesting to note that in both our and the Al Rashidi study, the carbonyls were sampled using DNPH-coated silica tubes, while Schubert used impingers with DNPH solution.

The results indicate that the chemistry is changing as puff duration varies. The humectant used in the shisha studied here is glycerol, a known source of volatile carbonyls and that has been well studied from electronic cigarettes. ${ }^{29-35}$ The thermal decomposition of the glycerol has many pathways, as was recently outlined by Jensen $e t a l .{ }^{35}$ It is likely that as the puff duration increases, the ratio of the different reaction pathways varies as well. At this time, it is not known what is causing the change in the chemistry of the thermal decomposition of glycerol as puff duration varies. Two possible reasons would be changes in temperature the shisha is exposed to and changes in oxygen content as the puff duration increases, revealing the need for future study. 


\section{CONCLUSION}

Puffing topography has an effect on the emissions from hookah. For particulate matter, the dominant topography factor is puff flow rate. For individual volatile carbonyl compounds, the emissions are affected by puff duration, with the concentration of formaldehyde increasing while others decrease at longer puff duration. The results presented are normalized by either puff volume (mass concentration) or total particulate matter (mass ratio) as this allows for comparison across topographies as well as providing a mechanism for determining emissions a user will be exposed to under real world settings. Puff-topuff variability in smoking topography means that knowing puff volume alone is insufficient to characterize emissions. For instance, particulate matter emitted per $\mathrm{mL}$ of air decreases over $40 \%$ from 150 to $250 \mathrm{~mL} \mathrm{sec}^{-1}$ puff flow rate. With the results presented here, and puffing topographies at the individual puff interval of hookah users, true emissions can be determined. Subsequent laboratory studies using multiple topographies will provide a better benchmark of user exposure, as well as what affect regulation will have on emissions from hookahs. In addition, taking topography into consideration will aid in closing the gap between emissions observed in the laboratory and greater emissions observed from users in the natural environment. ${ }^{17}$

It is essential to consider topography impacts on emissions when assessing regulations concerning additives, which may be permitted in waterpipe tobacco. In June 2018, the Food and Drug Administration issued an advance notice of proposed rulemaking to obtain information on how certain flavours attract youths to initiate tobacco product use. ${ }^{36}$ To understand the effect that flavour additives have on attracting youth, as well as the health effect of a given flavour additive, accurate emissions must be known. To obtain this information, two things will be required; natural environment topographies and collection of emissions in a laboratory setting using topographies that span the topographies observed. Failure to do this will result in an inaccurate picture of the health risk of each flavour additive. This is not just for the quantification of exposure to the given flavour compound, but other harmful and potentially harmful compounds as well. If for instance, a flavour additive influences a user to take slower, longer puffs, the results presented here indicate that the user will be exposed to more particulate matter, more nicotine and more formaldehyde.

\section{What this paper adds}

The puffing topographies of hookah users vary user-to-user and even puff-to-puff.

- To date, the majority of laboratory studies have analysed the emissions from hookah using only one puffing topography.

- This paper shows that emissions from hookah smoking are dependent on puffing topography with particulate matter production mainly influenced by puff flow rate and emission of volatile carbonyl compounds influenced by puff duration.

- One puffing topography may be insufficient to quantify the amount of emissions a user is exposed to; puffing profiles of an individual user's session should be used instead.

Contributors Study conception and design: NCE, ECH and RJR. Acquisition of data: NCE, SD, PK and AGD. Analysis and interpretation of data: NCE, ECH and RJR. Drafting of manuscript: NCE. Critical edits and revisions: NCE, ECH, RJR and AGD.
Funding This work was supported by NIDA/NIH and FDA CTP (1R01 DA04247001).

Disclaimer The content is solely the responsibility of the authors and does not necessarily represent the official views of the NIH or the FDA.

Competing interests None declared.

Patient consent for publication Not required.

Provenance and peer review Not commissioned; externally peer reviewed. Data availability statement Data are available upon reasonable request.

ORCID iD

Nathan C Eddingsaas http://orcid.org/0000-0003-1539-5415

\section{REFERENCES}

1 Salloum RG, Thrasher JF, Kates FR, et al. Water pipe tobacco smoking in the United States: findings from the National adult tobacco survey. Prev Med 2015;71:88-93.

2 Shihadeh A, Saleh R. Polycyclic aromatic hydrocarbons, carbon monoxide, "tar", and nicotine in the mainstream smoke aerosol of the narghile water pipe. Food Chem Toxicol 2005;43:655-61.

3 Al Rashidi M, Shihadeh A, Saliba NA. Volatile aldehydes in the mainstream smoke of the narghile waterpipe. Food Chem Toxicol 2008;46:3546-9.

4 Sepetdjian E, Shihadeh A, Saliba NA. Measurement of 16 polycyclic aromatic hydrocarbons in narghile waterpipe tobacco smoke. Food Chem Toxicol 2008:46:1582-90.

5 Daher N, Saleh R, Jaroudi E, et al. Comparison of carcinogen, carbon monoxide, and ultrafine particle emissions from narghile waterpipe and cigarette smoking: sidestream smoke measurements and assessment of second-hand smoke emission factors. Atmos Environ 2010;44:8-14.

6 Cobb CO, Shihadeh A, Weaver MF, et al. Waterpipe tobacco smoking and cigarette smoking: a direct comparison of toxicant exposure and subjective effects. Nicotine Tob Res 2011;13:78-87.

7 Schubert J, Heinke V, Bewersdorff J, et al. Waterpipe smoking: the role of humectants in the release of toxic carbonyls. Arch Toxicol 2012;86:1309-16.

8 Sepetdjian E, Abdul Halim R, Salman R, et al. Phenolic compounds in particles of mainstream waterpipe smoke. Nicotine Tob Res 2013;15:1107-12.

9 Schubert J, Hahn J, Dettbarn G, et al. Mainstream smoke of the waterpipe: does this environmental matrix reveal as significant source of toxic compounds? Toxicol Lett 2011;205:279-84.

10 Shihadeh A. Investigation of mainstream smoke aerosol of the argileh water pipe. Food Chem Toxicol 2003;41:143-52.

11 Shihadeh A, Azar S, Antonios C, et al. Towards a topographical model of narghile water-pipe café smoking: a pilot study in a high socioeconomic status neighborhood of Beirut, Lebanon. Pharmacol Biochem Behav 2004;79:75-82.

12 Maziak W, Rastam S, Shihadeh AL, et al. Nicotine exposure in daily waterpipe smokers and its relation to puff topography. Addict Behav 2011;36:397-9.

13 Blank MD, Brown KW, Goodman RJ, et al. An observational study of group waterpipe use in a natural environment. Nicotine Tob Res 2014;16:93-9.

14 Cobb CO, Blank MD, Morlett A, et al. Comparison of puff topography, toxicant exposure, and subjective effects in low- and high-frequency waterpipe users: a double-blind, placebo-control study. Nicotine Tob Res 2015;17:667-74.

15 Resently compleated study, results will be published at a later date.

16 Soule EK, Ramôa C, Eissenberg T, et al. Differences in puff topography, toxicant exposure, and subjective response between waterpipe tobacco smoking men and women. Exp Clin Psychopharmacol 2018:26:440-7.

17 Jawad M, Eissenberg T, Salman R, et al. Toxicant inhalation among singleton waterpipe tobacco users in natural settings. Tob Control 2019:28:181-8.

18 Hensel EC, Jayasekera S, Robinson RJ. Accounting for effects of system dynamics to improve accuracy of emissions reported in e-cig vaping machines. Inhal Toxicol 2018;30:343-53.

19 Robinson RJ, Eddingsaas NC, DiFrancesco AG, et al. A framework to investigate the impact of topography and product characteristics on electronic cigarette emissions. PLoS One 2018;13:e0206341.

20 Robinson RJ, Hensel EC, Al-Olayan AA, et al. Effect of e-liquid flavor on electronic cigarette topography and consumption behavior in a 2-week natural environment switching study. PLoS One 2018;13:e0196640.

21 Robinson RJ, Hensel EC, Roundtree KA, et al. Week long topography study of young adults using electronic cigarettes in their natural environment. PLOS One 2016;11:e0164038.

22 Robinson RJ, Hensel EC, Morabito PN, et al. Electronic cigarette topography in the natural environment. PLoS One 2015;10:e0129296.

23 Robinson RJ, Hensel EC, Morabito PN, et al. Framework to report total particulate mass and aldehyde emission characteristics from Hookah. SRNT 2019 Annual Meeting, San Francisco, CA, 2019.

24 Hensel EC, al Olayan AA, Sarles SE, et al. Hookah and Dual-Use behavior in the natural environment. 2018 Tobacco Regulatory Science Meeting, Bethesda, MD, 2018 


\section{Original research}

25 Eddingsaas NC, Hensel EC, DiFrancesco AG, et al. Effect of hookah hose dimensions and material on TPM, nicotine, and aldehydes. 2018 Tobacco Regulatory Science Meeting, Bethesda, MD, 2018.

26 Katurji M, Daher N, Sheheitli H, et al. Direct measurement of toxicants inhaled by water pipe users in the natural environment using a real-time in situ sampling technique. Inhal Toxicol 2010;22:1101-9.

27 Pagano T, DiFrancesco AG, Smith SB, et al. Determination of nicotine content and delivery in disposable electronic cigarettes available in the United States by gas chromatography-mass spectrometry. NICTOB 2016;18:700-7.

28 Shihadeh A, Salman R, Jaroudi E, et al. Does switching to a tobacco-free waterpipe product reduce toxicant intake? A crossover study comparing $\mathrm{CO}, \mathrm{NO}, \mathrm{PAH}$, volatile aldehydes, "tar" and nicotine yields. Food Chem Toxicol 2012;50:1494-8.

29 Conklin DJ, Ogunwale MA, Chen Y, et al. Electronic cigarette-generated aldehydes: the contribution of e-liquid components to their formation and the use of urinary aldehyde metabolites as biomarkers of exposure. Aerosol Science and Technology 2018;52:1219-32.
30 Qu Y, Kim K-H, Szulejko JE. The effect of flavor content in e-liquids on e-cigarette emissions of carbonyl compounds. Environ Res 2018;166:324-33.

31 El-Hellani A, Salman R, El-Hage R, et al. Nicotine and carbonyl emissions from popular electronic cigarette products: correlation to liquid composition and design characteristics. Nicotine Tob Res 2018;20:215-23.

32 Salamanca JC, Meehan-Atrash J, Vreeke S, et al. E-Cigarettes can emit formaldehyde at high levels under conditions that have been reported to be non-averse to users. Sci Rep 2018;8.

33 Klager S, Vallarino J, MacNaughton P, et al. Flavoring chemicals and aldehydes in e-cigarette emissions. Environ Sci Technol 2017:51:10806-13.

34 Wang P, Chen W, Liao J, et al. A Device-Independent evaluation of carbonyl emissions from heated electronic cigarette solvents. PLoS One 2017;12:e0169811.

35 Jensen RP, Strongin RM, Peyton DH. Solvent chemistry in the electronic cigarette reaction vessel. Sci Rep 2017;7.

36 Food and Drug Administration HHS. Prposed rule: regulation of flavors in tobacco products (83 Fr 12294). Wahington, DC; 2018. 